nephron

Practice
Nephron 2015;131:102-106

DOI: $10.1159 / 000439066$
Received: July 5, 2015

Accepted: July 21, 2015

Published online: September 5, 2015

\title{
Long Term Outcomes after Acute Kidney Injury: Lessons from the ARID Study
}

\author{
Kerry L. Horne ${ }^{a}$ Adam Shardlow ${ }^{a}$ Maarten W. Taala, b Nicholas M. Selby ${ }^{a}$ b \\ ${ }^{a}$ Department of Renal Medicine, Royal Derby Hospital, Derby, ${ }^{b}$ Division of Medical Science and Graduate Entry \\ Medicine, University of Nottingham, Nottingham, UK
}

\section{Key Words}

Acute kidney injury - E-alerts · Remote consent - Chronic kidney disease progression - Proteinuria $\cdot$ Albuminuria

\begin{abstract}
The high incidence and poor short-term outcomes of acute kidney injury (AKI) have focused attention on this global healthcare issue. Concurrently, the long-term effects of AKI are increasingly appreciated, namely, increased risk of subsequent chronic kidney disease, end stage kidney disease requiring renal replacement therapies and a higher rate of cardiovascular events. Whilst there is little doubt about the strength of these associations, knowledge gaps remain. To address some of these, the AKI Risk In Derby study commenced in 2013. This is a prospective case-control study investigating the long-term effects of AKI in a general hospitalized population (including those with less severe AKI). This review will summarize the background and rationale of this study, its design and methodology, as well as the 1-year outcome results from a preceding pilot study.
\end{abstract}

(C) 2015 S. Karger AG, Basel

\section{Does Acute Kidney Injury Lead to Chronic Kidney Disease?}

An increasingly held view is that of acute kidney injury (AKI) and chronic kidney disease (CKD) as interrelated syndromes, with AKI leading to $\mathrm{CKD}$ and $\mathrm{CKD}$ strongly predisposing to the development of AKI [1]. Whilst AKI and CKD share similar risk factors, the strength of their association is one of several arguments for the link between the two being causal $[2,3]$. A large number of clinical studies have shown AKI to increase the risk of both new-onset CKD [4] as well as hastening established CKD progression $[5,6]$. Concurrently, experimental data have revealed many similarities between the pathological processes that drive CKD progression and those that occur following an episode of AKI $[2,7]$. These mechanisms are multiple and varied, but are largely independent of the initial renal injury [7]. Of particular importance are maladaptive repair processes resulting in tubulointerstitial atrophy and fibrosis, which may arise because of pro-inflammatory signal preventing normal tubular cell regeneration/re-differentiation [7]. Alongside this, capillary rarefaction and vascular drop-out also occur, forming a vicious cycle and increasing the risk of subsequent ischemic injury [8].

However, much of the clinical data in this area are derived from interrogation of large administrative data sets. These data, based on clinical coding with inherent attribution errors, can risk confounding due to variation in the availability of follow-up measures of renal function. Administrative coding has been found to have

Contribution from the AKI \& CRRT 2015 Symposium at the 20th International Conference on Advances in Critical Care Nephrology, Manchester Grand Hyatt, San Diego, Calif., USA, February 17-20, 2015.

\section{KARGER 125}

(c) 2015 S. Karger AG, Base

$1660-8151 / 15 / 1312-0102 \$ 39.50 / 0$

E-Mail karger@karger.com

www.karger.com/nef
Dr. Nicholas M. Selby

Department of Renal Medicine, Royal Derby Hospital

Uttoxeter Road

Derby, DE22 3NE (UK)

E-Mail nicholas.selby@ nottingham.ac.uk 
a good positive predictive value for clinical diagnosis of AKI [9] but low sensitivity [10] and therefore underrepresents patients with less severe AKI. Furthermore, those studies with higher data resolution have tended to focus on AKI in specific circumstances such as in the context of coronary angiography [11], cardiac surgery [12] or dialysis requiring AKI (usually in an ICU setting) [6]. These clinical settings are not necessarily comparable with the majority of patients with AKI in the general hospital population and, in particular, the majority of patients who present with already established AKI from a community context [13]. These patients represent the greatest proportion of the overall AKI caseload and characteristically are older, have less severe AKI often resulting from mixed etiologies and are cared for in lower dependency settings [13]. Therefore, uncertainty exists as to the long-term sequelae of AKI in the extremes of age, the effects of the setting, severity and etiology of AKI and whether 'mild' AKI (including pre-renal azotemia) can impact on long-term outcomes. It is for these reasons that prospective studies are needed that include control groups and study a broad and representative population. Such studies also provide an opportunity to improve on early attempts to develop and validate methods to predict those patients at a higher risk of adverse long-term outcomes [4]. More evolved strategies would allow targeting of enhanced follow-up (and subsequent testing of interventions) to those with the greatest need, whilst providing reassurance to those with complete recovery and low risk of long-term effects. Such studies are now underway and include the AKI Risk In Derby (ARID) study in the UK [14], the focus of this review and the US-based Assessment Serial Evaluation and Subsequent Sequelae of Acute Kidney Injury (ASSESS-AKI) study [15]. These studies share common aims and similar approaches to delineate the long-term natural history of AKI and identify those factors that associate with the risk of long-term complications.

\section{Aims, Design and Methodology of ARID Study}

The ARID study is a prospective, observational casecontrol study that will determine the natural history of AKI in a general hospitalized population, including those with less severe AKI and pre-renal azotemia [14]. Study design is shown in figure 1. Change in renal function and CKD progression will be compared between AKI patients and matched controls at 3,12 and 36 months.
CKD progression will be defined as a fall in estimated glomerular filtration rate (eGFR) of $\geq 25 \%$ associated with a change in eGFR stage. Prevalence of proteinuria and albuminuria will be compared at each time point as will mortality (also tracked to 5 years), cardiovascular events and a composite renal end point of doubling of serum creatinine, commencement of renal replacement therapies or eGFR $<15 \mathrm{ml} / \mathrm{min} / 1.73 \mathrm{~m}^{2}$. Effects of setting and etiology of AKI will be studied, and factors associated with CKD progression determined with the aim of building prediction tools to stratify the risk of patients' long-term outcomes. The collection of stored samples will allow the testing of the additive value of novel biomarkers of renal injury and repair to clinical risk prediction models.

A novel feature of the ARID study is its use of automated screening and recruitment methodology. Cases and controls are identified from results of an electronic AKI detection system [13] following which participants are consented 'remotely'. The process of obtaining remote consent is well established in specialties such as gastroenterology and is particularly suited to situations where a large number of patients need to give consent for procedures that have low risks of complications [16]. This approach recognizes explicitly that consent is the process of appropriate information giving and decision making, and that the consent form is simply a record that this has taken place. The ARID protocol incorporates these principles as follows: comprehensive written information is sent to potential participants 3 months after their hospital admission, interested patients contact the research team via telephone, after confirming patient identity informed consent is obtained, a written consent form is then posted to the patient to sign and return to the research team. Pre-prepared sample packs are posted with the consent forms and patients then arrange tests at outpatient phlebotomy or local primary care services. Samples are handled separately from routine clinical samples with rapid transfer and analysis within $7 \mathrm{~h}$ in the central hospital laboratory. The aim was to create an efficient study capable of recruiting large numbers of patients, and this has been demonstrated by the completion of a pilot study that was performed to specifically test the novel aspects of the study design. Patient acceptability is high with ease and convenience of participating reported; pre-defined recruitment, withdrawal and matching criteria were also all achieved [14]. It may therefore be that this approach now has wider applicability for the design of other lowrisk, observational studies. 


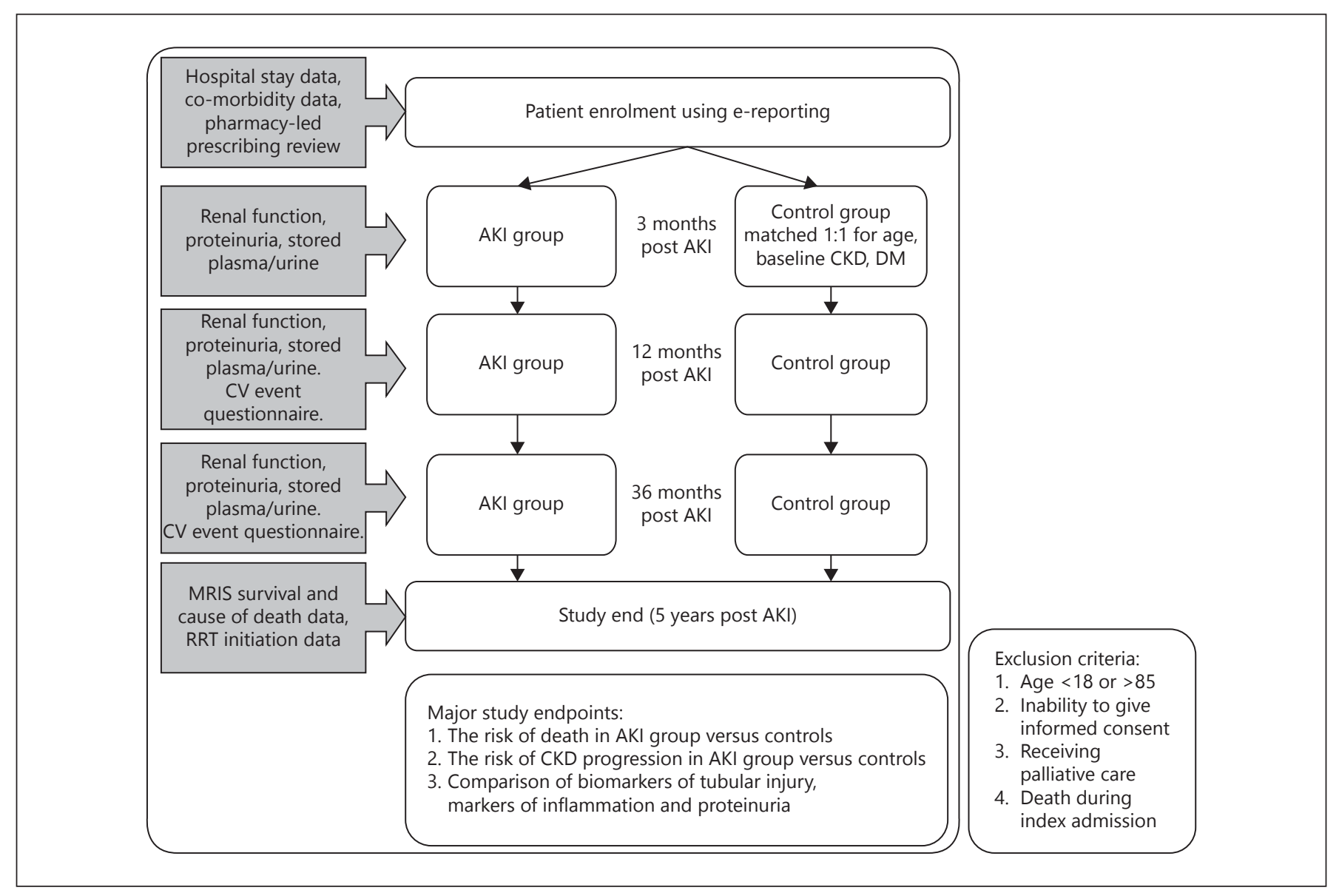

Fig. 1. ARID study design.

\section{Initial Results from ARID Pilot Study}

The pilot study included 300 matched participants, with no significant baseline differences in age or renal function [14]. The median age of patients who sustained AKI was 72 years and mean baseline eGFR was $67 \pm 21$ $\mathrm{ml} / \mathrm{min} / 1.73 \mathrm{~m}^{2}$, with $34 \%$ having pre-existing CKD. Consistent with clinical practice in general hospitalized populations, most (70\%) had mild AKI (AKI stage 1) of mixed etiology. Most obviously, findings from this initial work confirm the association between AKI and longterm decline in renal function. At both 3 and 12 months after hospitalization, AKI patients had higher serum creatinine and lower eGFR results as compared with controls. AKI patients also had a higher rate of CKD progression or development, and these differences were observed even with the significant proportion of 'mild' AKI. Despite this evidence of CKD progression, only a small proportion of patients $(1.3 \%)$ reached the composite renal end point by 1 year (doubling of serum creatinine, commencement of renal replacement therapies or eGFR $<15$ $\mathrm{ml} / \mathrm{min} / 1.73 \mathrm{~m}^{2}$ ).

Proteinuria was relatively common at 3 months after hospitalization, but to a much greater extent in patients who had sustained AKI. Seventy-one (50\%) patients in the AKI group had proteinuria at 3 months compared with $53(36 \%)$ controls $(\mathrm{p}=0.02)$, whilst corresponding figures for albuminuria were 48 (37\%) versus 30 (21\%), $\mathrm{p}=0.003$. In most cases, the degree of albuminuria/proteinuria was mild or moderate. At 12 months, the same patterns were observed although there was a general pattern of greater variability in the AKI group, with a trend to more patients showing a reduction of albuminuria and similar findings for resolution of albuminuria. Whilst these dynamic trends may suggest a causal role for AKI, these data cannot differentiate between pre-existing proteinuria increasing the risk of sustaining AKI versus a higher prevalence of proteinuria due to parenchymal in- 
jury sustained during an episode of AKI. We attempted to examine this further by contrasting these results with those from a prospective cohort study of 1,741 people with CKD that is also run at our center (the Renal Risk In Derby [RRID] study) [17], looking at those who sustained incidental AKI. In the RRID study, patients with CKD stage 3 submitted 3 consecutive early morning measurements of urinary ACR and PCR at baseline (i.e., before AKI episode) and then again at 12 months. One hundred three (5.9\%) participants had an episode of AKI in the first year of follow-up. Whilst overall albuminuria and proteinuria increased in participants with and without an episode of AKI, AKI was associated with a greater incidence of new-onset albuminuria (11 of 103 [11\%] vs. 71 of 1,518 [5\%], $\mathrm{p}=0.02$ ) but not new proteinuria ( 21 of 103 [20\%] vs. 282 of 1,518 [19\%], p = 0.7). Importantly, AKI was an independent risk factor for progression of albuminuria category over 1 year (in addition to age, diabetes and lower baseline eGFR) and for CKD progression. Therefore, albuminuria and proteinuria are increased after AKI but the increase in proteinuria appears to be more sustained. Taking these results together, they support the hypothesis that AKI may cause or exacerbate tubulointerstitial damage and contribute to the progression of CKD. It is interesting to speculate that the transient nature of the albuminuria in some AKI patients could reflect tubular injury followed by successful repair (or maladaptive repair in those with persistent/worsening albuminuria) [18]. Whilst albuminuria post-AKI may predict subsequent CKD progression [14], it remains to be determined how dynamic changes in albuminuria after AKI associate with risk of CKD progression.

\section{Opportunities for Improving Post-AKI Care}

Despite the increased risk of long-term renal dysfunction following AKI, it is important to state that AKI patients are divided into those with and without full recovery of renal function; that is, not all patients with AKI necessarily have adverse long-term outcomes [4]. A variety of independent associations with non-recovery and CKD progression following AKI have been described that include advanced age, severity of AKI, diabetes mellitus, preexisting $\mathrm{CKD}$, heart failure, increased Charlson comorbidity score and low serum albumin [19]. However, we do not yet have fully evolved and validated risk prediction scores that can be used in clinical practice to reliably identify those patients that require ongoing specialist care/follow-up and those that can be discharged to pri- mary care with low risk of long-term sequelae. Progress is this area is urgently required; it is clear that currently a large proportion of patients do not receive nephrology follow-up after AKI even when indicated [20]. There is also much work to be done to test the effectiveness of interventions in the post-AKI care; for example the role of RAAS blockade may be a double-edged sword, potentially benefiting those with significant proteinuria but possibly increasing risk of further AKI episodes. Whilst trials into specific interventions post-AKI are awaited, it would appear that there are a variety of immediate opportunities to improve basic care. This may include improved medication management (restarting important chronic medications that were stopped during AKI episode), advice on AKI prevention and improved clinical information about AKI in discharge documentation. Whilst we lack clear evidence on therapeutic interventions, it may be that quality improvement strategies have the potential to produce significant patient benefit within the shortest timeframe.

\section{Conclusions}

The debate as to whether AKI contributes to the onset and progression of CKD would appear to be won; an important next step is to elucidate which AKI patients recover fully with no long-term consequences and which do not. Only by describing these groups and then developing strategies for their recognition at an early time point can we move onward to formulating and testing strategies to ameliorate the long-term outcomes of AKI. Current prospective studies such as the ARID study will be the key to this process.

\section{Acknowledgments}

ARID study investigators: K.L. Horne, D. Moore, A. Akani, S. Shaw, R. Packington, H. Mahmoud, M.W. Taal and N.M. Selby.

References

1 Chawla LS, Eggers PW, Star RA, Kimmel PL: Acute kidney injury and chronic kidney disease as interconnected syndromes. N Engl J Med 2014;371:58-66.

2 Chawla LS, Kimmel PL: Acute kidney injury and chronic kidney disease: an integrated clinical syndrome. Kidney Int 2012;82:516524.

3 Coca SG, Singanamala S, Parikh CR: Chronic kidney disease after acute kidney injury: a systematic review and meta-analysis. Kidney Int 2012;81:442-448. 
4 Chawla LS, Amdur RL, Amodeo S, Kimmel PL, Palant CE: The severity of acute kidney injury predicts progression to chronic kidney disease. Kidney Int 2011;79:1361-1369.

5 Amdur RL, Chawla LS, Amodeo S, Kimmel PL, Palant CE: Outcomes following diagnosis of acute renal failure in U.S. Veterans: focus on acute tubular necrosis. Kidney Int 2009;76: 1089-1097.

6 Lo LJ, Go AS, Chertow GM, McCulloch CE, Fan D, Ordoñez JD, Hsu CY: Dialysis-requiring acute renal failure increases the risk of progressive chronic kidney disease. Kidney Int 2009;76:893-899.

7 Venkatachalam MA, Griffin KA, Lan R, Geng $\mathrm{H}$, Saikumar P, Bidani AK: Acute kidney injury: a springboard for progression in chronic kidney disease. Am J Physiol Renal Physiol 2010;298:F1078-F1094.

8 Basile DP, Donohoe D, Roethe K, Osborn JL: Renal ischemic injury results in permanent damage to peritubular capillaries and influences long-term function. Am J Physiol Renal Physiol 2001;281:F887-F899.

9 Tomlinson LA, Riding AM, Payne RA, Abel GA, Tomson CR, Wilkinson IB, Roland MO, Chaudhry AN: The accuracy of diagnostic coding for acute kidney injury in England - a single centre study. BMC Nephrol 2013;14: 58.

10 Vlasschaert ME, Bejaimal SA, Hackam DG, Quinn R, Cuerden MS, Oliver MJ, Iansavi- chus A, Sultan N, Mills A, Garg AX: Validity of administrative database coding for kidney disease: a systematic review. Am J Kidney Dis 2011;57:29-43.

11 James MT, Ghali WA, Tonelli M, Faris P, Knudtson ML, Pannu N, Klarenbach SW, Manns BJ, Hemmelgarn BR: Acute kidney injury following coronary angiography is associated with a long-term decline in kidney function. Kidney Int 2010;78:803-809.

12 Ishani A, Nelson D, Clothier B, Schult T, Nugent S, Greer N, Slinin Y, Ensrud KE: The magnitude of acute serum creatinine increase after cardiac surgery and the risk of chronic kidney disease, progression of kidney disease, and death. Arch Intern Med 2011;171:226233.

13 Selby NM, Crowley L, Fluck RJ, McIntyre CW, Monaghan J, Lawson N, Kolhe NV: Use of electronic results reporting to diagnose and monitor AKI in hospitalized patients. Clin J Am Soc Nephrol 2012;7:533-540.

14 Horne KL, Packington R, Monaghan J, Reilly T, McIntyre CW, Selby NM: The effects of acute kidney injury on long-term renal function and proteinuria in a general hospitalised population. Nephron Clin Pract 2014;128: 192-200.

15 Go AS, Parikh CR, Ikizler TA, Coca S, Siew ED, Chinchilli VM, Hsu CY, Garg AX, Zappitelli M, Liu KD, Reeves WB, Ghahramani N, Devarajan P, Faulkner GB, Tan TC, Kimmel
PL, Eggers P, Stokes JB; Assessment Serial Evaluation, and Subsequent Sequelae of Acute Kidney Injury Study Investigators: The assessment, serial evaluation, and subsequent sequelae of acute kidney injury (ASSESSAKI) study: design and methods. BMC Nephrol 2010;11:22.

16 British Society of Gastroenterology: Guidelines for postal consenting for outpatient endoscopic procedures. http://www.bsg.org.uk/ clinical-guidelines/endoscopy/guidelinesfor-postal-consenting-for-outpatientendoscopic-procedures.html.

17 McIntyre NJ, Fluck RJ, McIntyre CW, Taal MW: Risk profile in chronic kidney disease stage 3: older versus younger patients. Nephron Clin Pract 2011;119:c269-c276.

18 Dickson LE, Wagner MC, Sandoval RM, Molitoris BA: The proximal tubule and albuminuria: really! J Am Soc Nephrol 2014;25:443453.

19 Goldstein SL, Jaber BL, Faubel S, Chawla LS; Acute Kidney Injury Advisory Group of American Society of Nephrology: AKI transition of care: a potential opportunity to detect and prevent CKD. Clin J Am Soc Nephrol 2013;8:476-483.

20 Siew ED, Peterson JF, Eden SK, Hung AM, Speroff T, Ikizler TA, Matheny ME: Outpatient nephrology referral rates after acute kidney injury. J Am Soc Nephrol 2012;23:305312 . 